\title{
Frequency Scaling and Topology Comparison of Millimeter-wave CMOS VCOs
}

\author{
K. W. Tang ${ }^{+}$, S. Leung ${ }^{+}$, N. Tieu ${ }^{+}$, P. Schvan*, S. P. Voinigescu ${ }^{+}$ \\ ${ }^{+}$ECE Dept., University of Toronto, 10 King's College Rd. Toronto, ON, M5S 3G4, Canada \\ *NORTEL, 3500 Carling Av. Ottawa, ON, K2H 8E9, Canada
}

\begin{abstract}
This paper presents an algorithmic design methodology and frequency scaling technique for CMOS VCOs. It illustrates the almost ideal scaling of a fundamental $10-\mathbf{G H z}$, 90-nm CMOS Colpitts VCO with $-117 \mathrm{dBc} / \mathrm{Hz}$ phase noise and $4 \mathrm{dBm}$ output power to $77 \mathrm{GHz}$. The $77-\mathrm{GHz}$ VCO features linear $8.3 \%$ tuning range and a phase noise of $-100.3 \mathrm{dBc} / \mathrm{Hz}$ at $1 \mathrm{MHz}$ offset. The first complementary cross-coupled VCO operating at $77 \mathrm{GHz}$ is also reported. In addition, a new figure of merit for CMOS VCO based on the one defined in the 2003 ITRS is proposed to adequately account for the VCO output power and efficiency.
\end{abstract}

Keywords - Millimeter-wave, CMOS, VCO, frequency scaling, Colpitts, cross-coupled, figure of merit.

\section{INTRODUCTION}

SiGe HBT mm-wave VCOs have the advantage of large transistor voltage swings and low phase noise. However, their power dissipation is excessive. At lower frequencies, CMOS VCOs have gained popularity due to their low power dissipation brought about by the significantly lower supply voltages. However, to date, mm-wave CMOS VCOs suffer from inadequate output power levels, reduced tuning range and, until recently [1], from very poor phase noise. Here, a family of 90-nm CMOS VCOs with record tuning range and phase noise performance at $77 \mathrm{GHz}$ are presented.

A systematic design procedure for Colpitts VCOs implemented in SiGe HBT technology was presented in [2]. Similarly, in section II of this paper, an algorithmic design methodology for CMOS Colpitts and cross-coupled VCOs is described. Performance of each topology at RF and at mmwave frequencies is also discussed. A technique to scale the oscillation frequency $f_{\text {osc }}$ of these VCOs is covered in section III, while section IV explains the inadequacy of the VCO FoM as defined in the 2003 ITRS [3] and proposes a new FoM that adequately captures the power efficiency of VCOs in practical applications. Finally, measurement results are summarized in section $\mathrm{V}$.

\section{CMOS VCO Design Methodology}

\section{A. Colpitts $V C O$}

The schematic of the 77-GHz Colpitts VCO is shown in Fig. 1. The design methodology for the Colpitts VCO can be summarized as follows.

1) Determine $L_{\text {TANK }}$ according to the design constraints [2]. Choose smallest $\mathrm{L}_{\mathrm{TANK}}$ for minimum phase noise

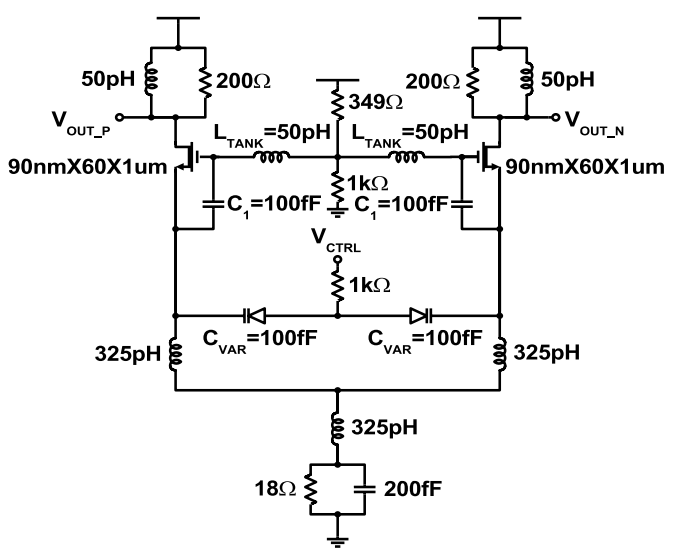

Figure 1. Schematic of 77-GHz Colpitts VCO.

and maximum tuning range, or largest $\mathrm{L}_{\mathrm{TANK}}$ for minimum power dissipation.

2) For a given $f_{\text {osc }}(1)$, the equivalent capacitance (2) is known once $\mathrm{L}_{\text {TANK }}$ is chosen. Select $\mathrm{C}_{1}>\mathrm{C}_{\mathrm{VAR}}$ for maximum tuning range, or $\mathrm{C}_{1}=\mathrm{C}_{\mathrm{VAR}}$ for minimum power dissipation.

$$
\begin{gathered}
f_{\text {osc }}=\frac{1}{2 \pi \sqrt{L_{T A N K} C_{e q}}} \\
C_{e q}=\frac{\left(C_{1}+C_{G S}\right)\left(C_{V A R}+C_{S B}\right)}{C_{1}+C_{V A R}+C_{G S}+C_{S B}}+C_{G D} \approx \frac{C_{1} C_{V A R}}{C_{1}+C_{V A R}}
\end{gathered}
$$

3) Bias the transistors at the optimum noise figure current density of $0.15 \mathrm{~mA} / \mu \mathrm{m}$ [4] for minimum phase noise. Size transistors (gate width W) to provide enough negative resistance for oscillation (3).

$$
\begin{gathered}
G_{m} \geq \omega_{o s c}{ }^{2} C_{1} C_{V A R} R_{S} \\
R_{S}=\frac{\omega_{o s c} L_{T A N K}}{Q_{L}}
\end{gathered}
$$

\section{B. Cross-coupled VCO}

The typical schematic of a cross-coupled VCO is shown in Fig. 2. Its design methodology can be summarized as follows.

1) Choose smallest $L_{\text {TANK }}$ for maximum tuning range, or largest $\mathrm{L}_{\text {TANK }}$ for minimum power dissipation. 


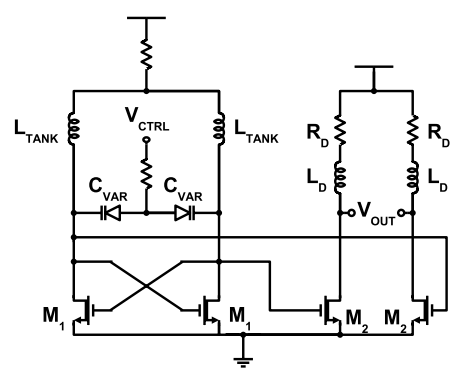

Figure 2. Schematic of cross-coupled VCO.

2) For given oscillation frequency (1), the equivalent capacitance (5) is known once $\mathrm{L}_{\text {TANK }}$ is chosen. In contrast to the Colpitts topology, $C_{e q}$ is heavily dependent on the parasitic capacitance of the transistor.

$C_{e q}=C_{V A R}+C_{G S}+4 C_{G D}+C_{D B}+C_{G S_{-} M_{2}}+C_{G D_{-} M_{2}}$

3) Bias the transistors at $0.15 \mathrm{~mA} / \mu \mathrm{m}$ for minimum phase noise. Determine transistor size from oscillation condition (6).

$$
\begin{gathered}
\left(G_{m} R_{P}\right)^{2} \geq 1 \\
R_{P}=\omega_{o s c} L_{T A N K} Q_{L}
\end{gathered}
$$

4) Subtract the parasitic capacitances of the transistor from the equivalent capacitance to obtain the varactor size.

In cross-coupled VCOs, as the parasitic capacitances of transistors are directly lumped to the tank capacitance, the tuning range is limited by the transistor size. It is further degraded by the output buffer which is always present in crosscoupled VCOs, either to drive the $50-\Omega$ test equipment or to distribute the VCO signal to mixers and frequency dividers in an IC. At mm-wave frequencies, where a relatively large transistor size has to be used to maintain oscillation, tuning range is sacrificed. At lower frequencies, large tuning range can be realized with small transistor and large varactor sizes which also leads to lower power dissipation, and thus to a superior figure of merit. However, for the same reasons, its output power is generally far below the acceptable range of 0 to $5 \mathrm{dBm}$. By comparison, the Colpitts VCO requires larger transistor size and current to produce the oscillation, and hence dissipates more power. Nevertheless, since the output buffer is built into the topology, its power efficiency and output power are higher. Moreover, its tuning range is mainly determined by the $C_{l}: C_{V A R}$ ratio, which is independent of the oscillation frequency. Most importantly, $f_{o s c}$ depends primarily on the passive components $\left(L_{T A N K}, C_{l}\right.$ and $\left.C_{V A R}\right)$ which implies that redesigning the VCO to another frequency can be achieved simply by scaling the passive components of the VCO.

\section{FREQUENCY SCALING}

From eqns. (1) and (2) in the previous section, if $L_{T A N K}, C_{I}$ and $C_{V A R}$ are all reduced by a factor of $k$, the oscillation frequency of a Colpitts VCO will be scaled by the same factor:

$$
\begin{gathered}
L_{\text {TANK }}{ }^{\prime}=\frac{L_{\text {TANK }}}{k}, C_{1}{ }^{\prime}=\frac{C_{1}}{k}, C_{V A R}{ }^{\prime}=\frac{C_{V A R}}{k} \\
f_{\text {osc }}{ }^{\prime}=k f_{\text {osc }}
\end{gathered}
$$

If the series resistance of the tank inductor is also scaled down with the tank inductance:

$$
R_{S}^{\prime}=\frac{R_{S}}{k}
$$

Substituting (10) into (3), we obtain:

$$
G_{m}{ }^{\prime}=\frac{G_{m}}{k}
$$

which implies that the transistor size and bias current, can be reduced by the same factor. Note that $f_{\text {osc }}$ scales exactly even if the parasitic capacitance of the transistor is taken into account. However, $R_{S}$ does not scale linearly as $f_{\text {osc }}$ increases because it includes the parasitic gate and source resistances of the transistor, both of which increase as the total gate width is reduced. As a result, when a VCO design is scaled to a very high frequency, a relatively larger transistor is required to maintain oscillation than that given by (11). Furthermore, it is critical to scale the entire VCO layout, i.e. transistor layout, component orientation, as well as the interconnect routing between components to ensure that layout parasitics also scale.

\section{CMOS VCO FIGURE OF MERIT}

According to the 2003 ITRS, the FoM for VCOs is defined as:

$$
F o M_{1}=\left(\frac{f_{\text {osc }}}{\Delta f}\right)^{2} \frac{1}{L[\Delta f] P_{\text {diss }}}
$$

However, (12) does not include the output power generated by the VCO. As a result, it artificially rewards designs with low DC power and mediocre phase noise, rather than promoting low phase noise with high power efficiency. It also leads to the misleading conclusion that the cross-coupled topology is superior to the Colpitts one in CMOS technology [5] and that current CMOS mm-wave VCOs actually outperform SiGe HBT VCOs. In practical applications, VCO output power is critical to drive mixers, buffers and power amplifiers. For example, a $77-\mathrm{GHz}$ automotive radar transmitter needs to generate in excess of $+16 \mathrm{dBm}$ in a $50-\Omega$ impedance. This means that in a CMOS system implementation, the design burden is simply shifted from the VCO to the PA. Therefore, it is important to include output power in the VCO FoM:

$$
\mathrm{FoM}_{2}=\left(\frac{f_{\text {osc }}}{\Delta f}\right)^{2} \frac{P_{\text {out }}}{L[\Delta f] P_{\text {diss }}}
$$

\section{Measurement Results}

To validate the above observations, $12 \mathrm{VCO}$ test structures have been fabricated in TSMC's $180-\mathrm{nm}$ and STM's $90-\mathrm{nm}$ CMOS technologies. Both cross-coupled and Colpitts 


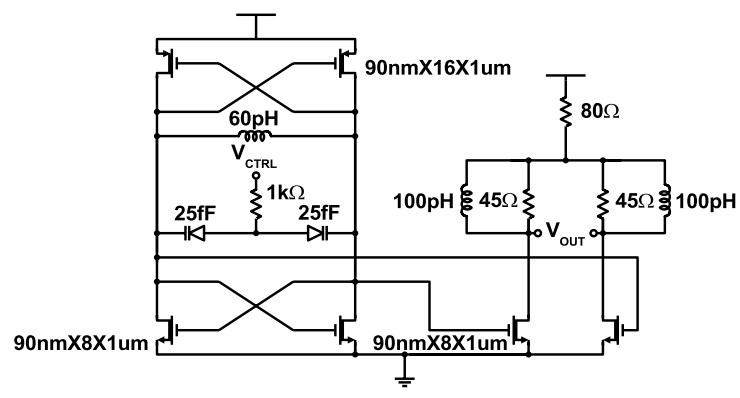

Figure 3. Schematic of 77-GHz cross-coupled VCO.

topologies were fabricated at 10,20,40,50 and $77 \mathrm{GHz}$. In all cases, the MOSFETs were biased at the optimum noise figure current density. The schematic of the 77-GHz CMOS crosscoupled VCO is shown in Fig. 3. Its measured output power, phase noise and tuning characteristics are shown in Fig. 4. It can be tuned between 76.2 and $78.2 \mathrm{GHz}$, with a measured phase noise of $-84.3 \mathrm{dBc} / \mathrm{Hz}$ at $10 \mathrm{MHz}$ offset. The core transistors dissipate between $5 \mathrm{~mA}$ and $7.5 \mathrm{~mA}$ when the supply varies between $1.5 \mathrm{~V}$ and $1.8 \mathrm{~V}$. This marks the first demonstration of a circuit with p-MOSFETs operating at $77 \mathrm{GHz}$.

As illustrated in Fig. 5, the 77-GHz Colpitts VCO can be tuned between 73.8 and $80 \mathrm{GHz}$, with a measured phase noise as low as $-100.3 \mathrm{dBc} / \mathrm{Hz}$ at $1 \mathrm{MHz}$ offset (Fig. 6). It draws 18 to $25 \mathrm{~mA}$ from $1.2 \mathrm{~V}$ to $1.5 \mathrm{~V}$ supply. It is a scaled replica of a $10-\mathrm{GHz}$ Colpitts VCO $\left(L_{T A N K}=435 \mathrm{pH}, C_{l}=C_{V A R}=\right.$ $800 \mathrm{fF}, 100 \mathrm{x} 1 \mathrm{um}$ transistor size). The measured performance of the latter is shown in Fig. 7, with $-117 \mathrm{dBc} / \mathrm{Hz}$ at $1 \mathrm{MHz}$ offset when biased from 1.2 V. For the purpose of comparison, a $10-\mathrm{GHz}$ NMOS cross-coupled VCO $\left(M_{1}=M_{2}=24 \times 1 \mathrm{um}\right.$, $\left.L_{T A N K}=435 \mathrm{pH}, C_{V A R}=260 \mathrm{fF}\right)$ was also fabricated, and its performance is shown in Fig. 8. It can be tuned between 9.3 and $10.9 \mathrm{GHz}$, with a phase noise of $-109.2 \mathrm{dBc} / \mathrm{Hz}$ at $1 \mathrm{MHz}$ offset. The die photo of the 77-GHz Colpitts VCO is shown in Fig. 9. The VCO core occupies $0.22 \mathrm{~mm}$ x $0.16 \mathrm{~mm}$.

A comparison between W-band CMOS and SiGe HBT VCOs using $\mathrm{FoM}_{1}$ and $\mathrm{FoM}_{2}$ is shown in Figs. 10 and 11, respectively. Based on $\mathrm{FoM}_{2}$, it becomes apparent that $\mathrm{SiGe}$ HBT VCOs exhibit superior performance to CMOS VCOs at $77 \mathrm{GHz}$. Table I summarizes state-of-the-art CMOS and SiGe HBT VCOs in the $60-\mathrm{GHz}$ to $120-\mathrm{GHz}$ range. The FoM of the

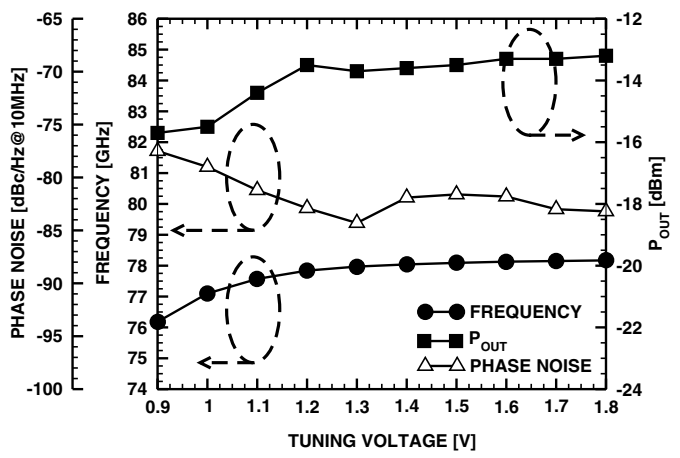

Figure 4. Frequency, output power and phase noise versus tuning voltage of the $77-\mathrm{GHz}$ cross-coupled $\mathrm{VCO}$.

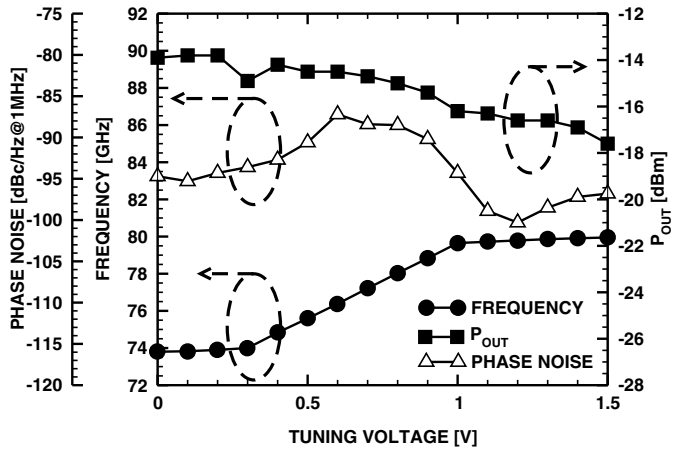

Figure 5. Frequency, output power and phase noise versus tuning voltage of the 77-GHz Colpitts VCO.

77-GHz Colpitts VCO is almost identical to that of its $10-\mathrm{GHz}$ replica, indicating almost perfect scaling of oscillation frequency and phase noise. For the $10-\mathrm{GHz}$ VCOs described in this work, the Colpitts topology achieves better figures of merit than the cross-coupled topology, despite the fact that its power dissipation is four times larger. Furthermore, its $\mathrm{FoM}_{2}$, output power, and phase noise are comparable to those of the best SiGe HBT VCOs, indicating that CMOS VCOs are capable of delivering similar performance to the SiGe HBT ones at lower frequencies.

\section{CONCLUSION}

An algorithmic design methodology and frequency scaling technique for CMOS VCOs has been presented and validated in measurements. Record phase noise and tuning range have been achieved at $77 \mathrm{GHz}$ using a Colpitts topology, and the first cross-coupled VCO employing p-MOSFETs operating above $50 \mathrm{GHz}$, has also been demonstrated. Finally, a new figure of merit has been proposed to allow for a realistic comparison between VCO topologies and technologies. While the phase noise of CMOS VCOs at mm-waves is now comparable to that of SiGe HBT ones, they continue to underperform in terms of output power levels.

\section{ACKNOWLEDGMENT}

We would like to thank Dr. M. T. Yang for his support and access to 180 -nm CMOS. This work was funded by NORTEL. Fabrication was provided by TSMC and through NORTEL and CMC by STMicroelectronics. We thank CMC for CAD tools, $\mathrm{CFI}$ and OIT for test equipment.

\section{REFERENCES}

[1] D. Huang, et al., "A $60 \mathrm{GHz}$ CMOS VCO Using On-Chip Resonator with Embedded Artificial Dielectric for Side, Loss and Noise Reduction," ISSCC Digest, pp. 314-315, Feb 2006.

[2] C. Lee, et al., "SiGe BiCMOS 65-GHz BPSK Transmitter and 30 to 122 GHz LC-Varactor VCOs with up to $21 \%$ Tuning Range," IEEE CSICS, pp. 179-182, Oct 2004.

[3] International Technology Roadmap for Semiconductors - System Drivers (2003 Edition). [Online] Avaliable: http://www.itrs.net/Links/ 2003ITRS/SysDrivers2003.pdf.

[4] S. P. Voinigescu, et al., "RF and Millimeter-Wave IC Design in the Nano-(Bi)CMOS Era," in Si- based Semiconductor Components for 


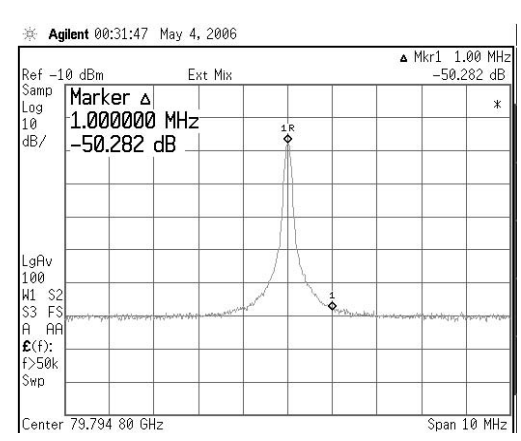

Figure 6. Averaged (100 sweeps) spectral plot of phase noise in 77-GHz Colpitts VCO, showing-100.3dBc/Hz@1 MHz offset.

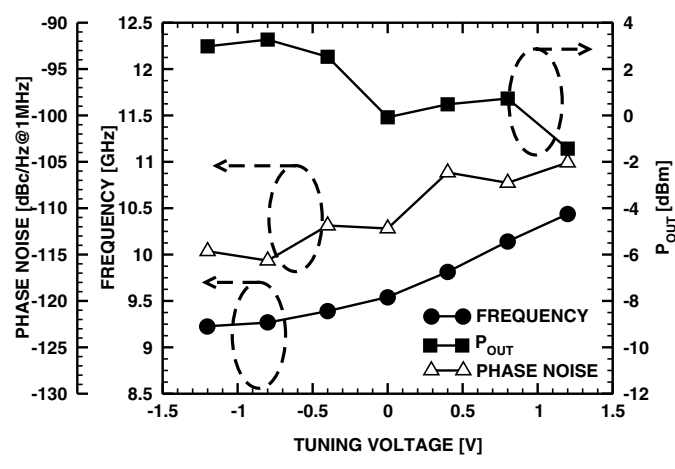

Figure 7. Frequency, output power and phase noise versus tuning voltage of the $10-\mathrm{GHz}$ Colpitts VCO.

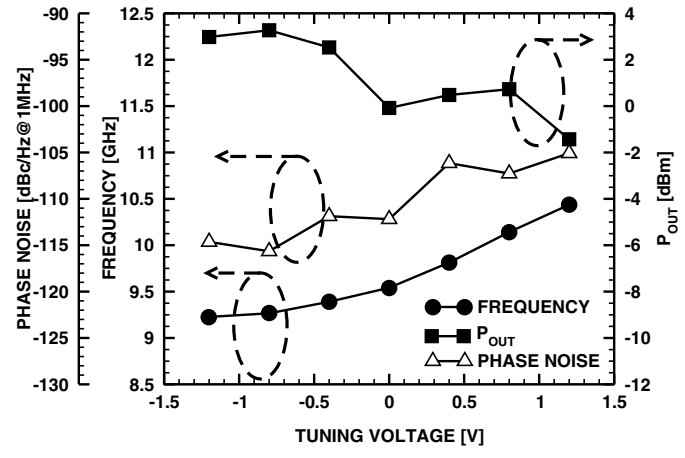

Figure 8. Frequency, output power and phase noise versus tuning voltage of the $10-\mathrm{GHz}$ cross-coupled $\mathrm{VCO}$.

Radio-Frequency Integrated Circuits, pp.33-62, Transworld Research Network, 2006. ISBN:81-7895-196-7.

[5] P. Andreani, et al., "A Study of Phase Noise in Colpitts and LC-Tank CMOS Oscillators," IEEE JSSC, May 2005.

[6] Nicolson, S., et al., "Design and Scaling of SiGe BiCMOS VCOs Operating Near 100GHz," BCTM 2006, in press.

[7] H. Li, et al., "Fully Integrated SiGe VCOs with Powerful Output Buffer for 77-GHz Automotive Radar Systems and Applications Around 100 GHz,” IEEE JSSC, vol. 39, pp. 1650-1658, Oct. 2004.

[8] R. Wanner, et al., "A SiGe Monolithically Integrated 75 GHz Push-Push VCO," Si Monolithic ICs in RF Sys. Digest, pp. 375-378, 2005

[9] W. Perndl, et al., "Voltage-Controlled Oscillators up to $98 \mathrm{GHz}$ in $\mathrm{SiGe}$

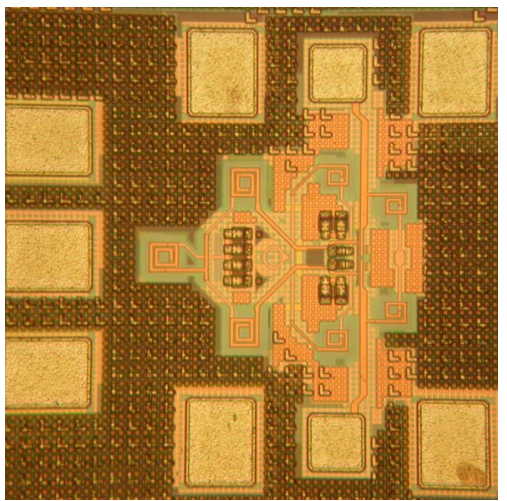

Figure 9. Die photo of 77-GHz Colpitts VCO.

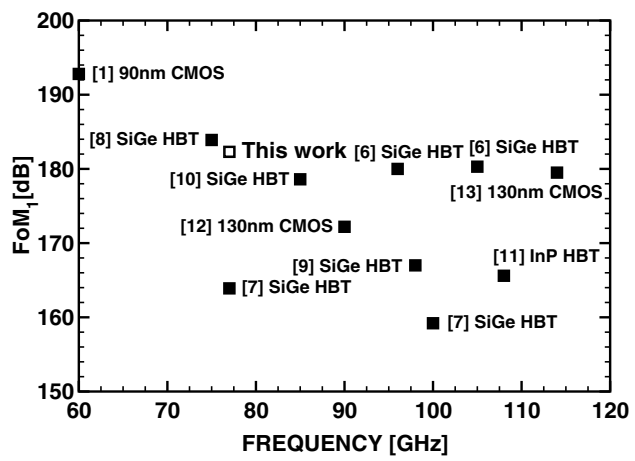

Figure 10. $\mathrm{FoM}_{1}$ of the state-of-the-art SiGe HBT and CMOS VCOs.
Bipolar Technology,” IEEE JSSC, Oct. 2004.

[10] B.A. Floyd, "V-band and W-band SiGe Bipolar Low-noise Amplifier and Voltage-Controlled Oscillators," RFIC Symposium Digest, pp. 295298, June 2004.

[11] Kobayashi, K. W., et al., "A 108-GHz InP-HBT monolithic push-push VCO with low phase noise and wide tuning bandwidth," IEEE JSSC, vol. 34, no. 9, pp. 1225-1232, Sept. 1999.

[12] C. Cao, et al., "A 90-GHz Voltage-Controlled Oscillator with a 2.2-GHz Tuning Range in a 130-nm CMOS Technology," Sym. on VLSI Cir. Digest, pp. 242-243, Jun 2005.

[13] Huang, P., et al., "A $114 \mathrm{GHz}$ VCO in 0.13 um CMOS technology," ISSCC Digest, pp. 404-406, Feb 2005.

TABLE I. PERFORMANCE SUMMARY OF THIS WORK AND STATE-OF-THE-ART SiGE HBT AND CMOS VCOS.

\begin{tabular}{|c|c|c|c|c|c|c|c|c|}
\hline Reference & Process & $\begin{array}{c}\mathbf{f}_{\text {osc }} \\
{[\mathbf{G H z}]}\end{array}$ & $\begin{array}{c}\text { Tuning } \\
{[\%]}\end{array}$ & $\begin{array}{c}\text { Phase Noise } \\
\text { [dBc/Hz] }\end{array}$ & $\begin{array}{c}\mathbf{P}_{\text {out }} \\
{[\mathbf{d B m}]}\end{array}$ & $\begin{array}{c}\mathbf{P}_{\text {diss }} \\
{[\mathbf{m W}]}\end{array}$ & $\begin{array}{l}\text { FoM }_{1} \\
{[\mathrm{~dB}]}\end{array}$ & $\begin{array}{c}\text { FoM }_{2} \\
{[\mathrm{~dB}]}\end{array}$ \\
\hline \multirow[t]{2}{*}{ This work - Colpitts } & \multirow[t]{2}{*}{ 90nm CMOS } & 10 & 12.2 & -117.5@1MHz & 4.0 & 36 & 181.9 & 185.9 \\
\hline & & 77 & 8.1 & $-100.3 @ 1 \mathrm{MHz}$ & -13.8 & 37.5 & 182.3 & 168.5 \\
\hline This work - NMOS cross-coupled & 90nm CMOS & 10 & 15.8 & -109.2@1MHz & -2.2 & 7.5 & 180.4 & 178.2 \\
\hline This work - CMOS cross-coupled & 90nm CMOS & 77 & 2.6 & -84.3@10MHz & -13.2 & 13.5 & 150.7 & 137.5 \\
\hline [1] & 90nm CMOS & 60 & 0.17 & $-100 @ 1 \mathrm{MHz}$ & -23.2 & 1.9 & 192.8 & 169.6 \\
\hline \multirow[t]{2}{*}{ [6] } & SiGe HBT, $\mathrm{f}_{\mathrm{T}}=170 \mathrm{GHz}$ & 96 & 4.6 & -101.6@1MHz & 0.7 & 133 & 180.0 & 180.7 \\
\hline & SiGe HBT, $\mathrm{f}_{\mathrm{T}}=230 \mathrm{GHz}$ & 105 & 4.4 & -101.3@1MHz & 2.5 & 133 & 180.3 & 183.0 \\
\hline \multirow[t]{2}{*}{ [7] } & \multirow[t]{2}{*}{ SiGe HBT, $f_{\mathrm{T}}=175 \mathrm{GHz}$} & 77 & 8.7 & -97@1MHz & 18.5 & 1200 & 163.9 & 182.4 \\
\hline & & 100 & 6.2 & -90@1MHz & 14.3 & 1200 & 159.2 & 173.5 \\
\hline [8] & SiGe HBT, $\mathrm{f}_{\mathrm{T}}=200 \mathrm{GHz}$ & 75 & 6.1 & $-105 @ 1 \mathrm{MHz}$ & 3.5 & 72 & 183.9 & 187.4 \\
\hline [9] & SiGe HBT, $f_{\mathrm{T}}=200 \mathrm{GHz}$ & 98 & 3.3 & -85@1MHz & -6 & 60 & 167.0 & 161.0 \\
\hline [10] & SiGe HBT, $f_{\mathrm{T}}=200 \mathrm{GHz}$ & 85 & 2.7 & -94@1MHz & -8 & 25 & 178.6 & 170.6 \\
\hline$[11]$ & $\mathrm{InP} H B T, \mathrm{f}_{\mathrm{T}}=75 \mathrm{GHz}$ & 108 & 2.6 & -88@1MHz & 0.92 & 204 & 165.6 & 166.5 \\
\hline$[12]$ & 130nm CMOS & 90 & 2.4 & $-105 @ 10 \mathrm{MHz}$ & -16 & 15.5 & 172.2 & 156.2 \\
\hline [13] & 130nm CMOS & 114 & 2.1 & -107.6@10MHz & -22.5 & 8.4 & 179.5 & 157.0 \\
\hline
\end{tabular}

